\title{
VARIAÇÃO ESPAÇO-TEMPORAL DO MEROPLÂNCTON NO ESTUÁRIO DO RIO PIRAQUÊ-AÇÚ, ARACRUZ, ESPÍRITO SANTO*
}

\author{
MAGRIS, R. A. ${ }^{1} \&$ LOUREIRO FERNANDES, L. ${ }^{2}$ \\ ${ }^{1}$ Bolsista CNPq. E-mail: ra_magris@yahoo.com.br \\ ${ }^{2}$ Universidade Federal do Espírito Santo - Depto. de Ecologia e Recursos Naturais - Av. Fernando \\ Ferrari s/n - CEP29060-900 - Vitória, ES.
}

\begin{abstract}
Magris, R.A. \& Loureiro Fernandes, L. 2005. Meroplankton spatial and temporal variability at the Piraquêaçú river estuary, Aracruz, Espírito Santo State. Braz. J. Aquat. Sci. Technol. 9(1):55-60. ISSN 1808-7035. Meroplankton was studied at the Piraquê-açú River Estuary, city of Aracruz, State of Espírito Santo, with the aim to understand their temporal and spatial distribution in the environment. Samples were collected monthly (between May and December 2003) in four sampling locations distributed along the estuary, using a conical plankton net with a $50 \mathrm{~cm}$ mouth opening and 200 micron mesh size. The samples were obtained using the net of the plankton. Temperature and salinity were measured in situ. Temperature showed higher variation between the months and salinity between the locations. The main taxonomic groups found were Cirripedia, Decapoda, Stomatopoda, Gastropoda, Bivalvia, Polychaeta, Echinodermata and Bryozoa. The most abundant groups were represented by Brachyura (mainly Uca burgersi, Eurytium limosum, Pachygrapsus transversus, Metasesarma rubripes and Sesarma rectum), Paguridae (Pagurus genus) and Diogenidae, Caridea (Alpheidae family), Cirripedia and Gastropoda. For the majority of the taxa there was significant differences between months, but only Cirripedia, Ophiuroidea and Echinoidea showed significant differences between sampling locations. The taxa found were grouped according to their abundance variation in the different months, what may reflect different life cycle strategies among species.
\end{abstract}

Keywords: meroplankton, larvae, distribution, Piraquê-açú, Espírito Santo.

\section{INTRODUÇÃO}

O meroplâncton pode constituir uma importante fração da biomassa zooplanctônica nos ambientes estuarinos em certos períodos do ano (Kennish \& Lutz, 1995). Esta comunidade é formada por larvas de espécies neríticas e costeiras, que aproveitam a intrusão de água salgada para penetrar no estuário; larvas de espécies estuarinas que migram deste ambiente para a zona marinha e só retornam ao estuário nos estágios finais da fase planctônica; e ainda espécies estuarinas que completam todo o desenvolvimento larval dentro do próprio estuário (Drake et al., 1998).

A dinâmica do meroplâncton representa um importante papel na ecologia e na evolução das espécies marinhas (Kennish \& Lutz, 1995). O levantamento taxonômico e a compreensão da distribuição espacial e temporal destas larvas no ambiente são importantes já que muitos dos seus componentes possuem interesse pesqueiro (e.g. larvas de diversos crustáceos e moluscos) (Brandini et al., 1997). Estes estudos contribuem também com o processo de conservação da biodiversidade; manejo e uso sustentável dos recursos naturais marinhos; e estabelecem possíveis relações

"Trabalho financiado pelo Projeto RECOS - Instituto do Milênio MCT/CNPq/PADCTIII da biota com as características físicas e químicas do ambiente, incluindo a interferência humana como fator alterador das condições naturais (Omori \& Ikeda, 1992).

Este trabalho tem como objetivo estudar a distribuição espaço-temporal das larvas meroplanctônicas no estuário do rio Piraquê-açú, sendo este um dos primeiros trabalhos deste gênero para o estado do Espírito Santo.

\section{MATERIAL E MÉTODOS}

O estuário do rio Piraquê-açú está localizado no distrito de Santa Cruz (4009'W e $19^{\circ} 57^{\prime}$ S), município de Aracruz, Espírito Santo, possuindo um manguezal que ocupa uma área de $12.3 \mathrm{~km}^{2}$.

As coletas de meroplâncton foram realizadas mensalmente (entre maio e dezembro de 2003), a cada ciclo de maré de quadratura, em quatro pontos amostrais distribuídos ao longo do estuário (Figura 1). Arrastos horizontais subsuperficiais foram feitos utilizando-se uma rede de plâncton cônica de $50 \mathrm{~cm}$ de boca e malha de 200 micrômetros, dotada de fluxômetro mecânico para o cálculo do volume de água filtrada pela rede. O material coletado foi fixado em solução aquosa de formalina 5\% tamponada com tetraborato de sódio. 


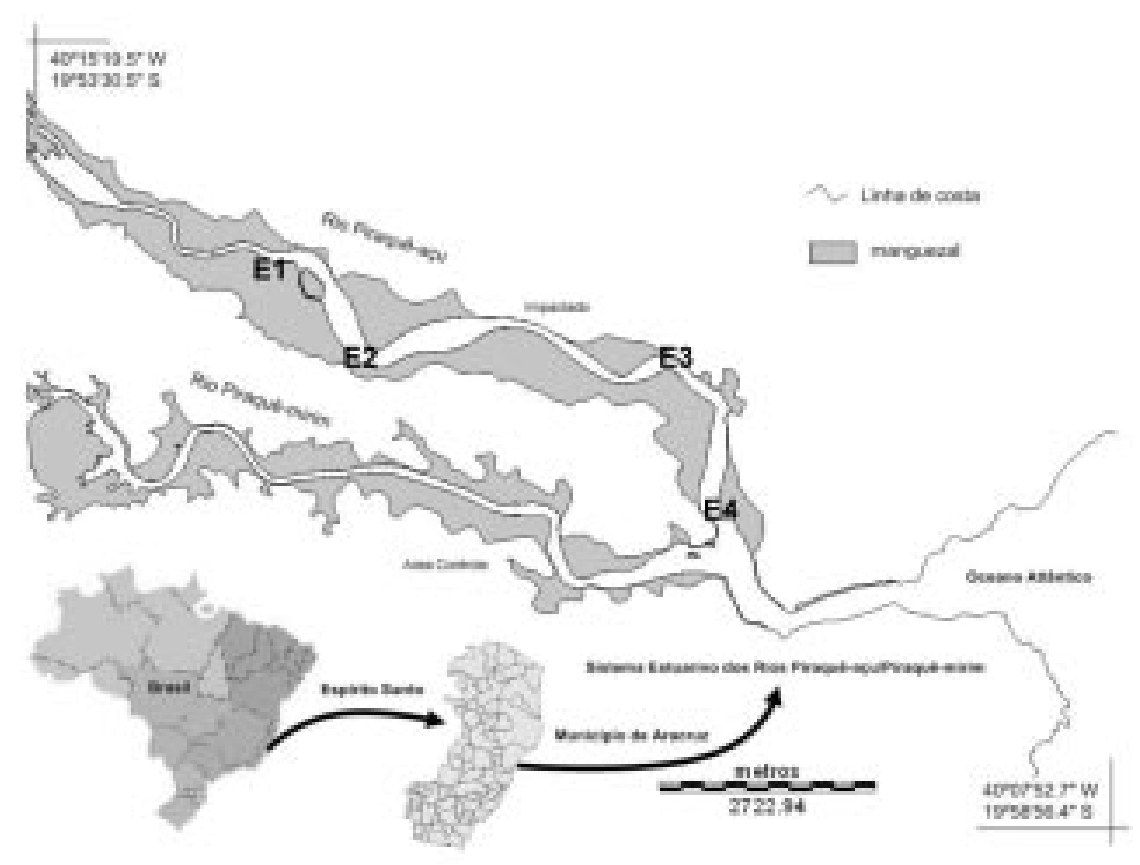

Figura 1 - Mapa da região de estudo incluindo os pontos amostrais E1 a E4 nos estuário do rio Piraquê-açú.

Temperatura e salinidade foram obtidas in situ utilizando um multiparâmetro portátil YSI85.

O meroplâncton foi quantificado e identificado até o menor nível taxonômico possível utilizando literatura pertinente (Boschi, 1981 e 1996; Pohle et al., 1999; Smith \& Johnson, 1997; Gurney, 1942).

Foram determinados os valores de abundância dos taxa encontrados nas amostras e estes normalizados com log-transformação utilizando-se a fórmula de Bray-Curtis. Foram efetuadas ANOVAS do tipo III sobre as densidades dos taxa (variáveis dependentes) para testar as diferenças entre meses (padrão temporal) e pontos (padrão espacial). A homogeneidade das variâncias entre os meses de coleta e os pontos amostrais foi verificada através do teste de Duncan. Foi realizada a análise Multivariada de Conglomerados (Cluster) pelo método de UPGMA utilizando a medida de Chord para verificar o grau de similaridade entre os taxa.

\section{RESULTADOS E DISCUSSÃO}

No estuário do rio Piraquê-açú, a temperatura máxima atingida foi de $26,7^{\circ} \mathrm{C}$ em dezembro e a mínima de $23,2^{\circ} \mathrm{C}$ em julho, com média anual de $25^{\circ} \mathrm{C}$ (Figura 2). Não foram observadas grandes variações de temperatura entre os pontos de amostragem. Em relação à salinidade, as maiores variações foram observa- das entre os pontos amostrais. O valor máximo obtido foi de 37,4 no ponto E4 localizado mais próximo à desembocadura e o mínimo de 24,6 no ponto E1, mais à montante, sendo a média entre pontos de amostragem de 33,5 (Figura 3). O mesmo padrão temporal para temperatura e espacial para a salinidade já foi verificado por Sterza (2002) na baía de Vitória e por Lopes (1996) na baía de Paranaguá.

Os principais grupos taxonômicos encontrados foram Cirripedia, Decapoda, Stomatopoda, Gastropoda, Bivalvia, Polychaeta, Echinodermata e Bryozoa. Entre estes, as larvas de Decapoda, de Cirripedia e de Gastropoda se destacaram pela dominância e ocorrência nas amostras. Estes grupos perfizeram uma média superior a $80 \%$ das larvas encontradas, o que mostra a forte influência destes taxa sobre o padrão geral de distribuição do meroplâncton (Figuras 4 e 5).

Os náuplios de Cirripedia foram abundantes em todos os meses de coleta, aparentando adotar a estratégia reprodutiva mais oportunista-generalista, caracterizada pela grande alocação de energia para os eventos reprodutivos e de grande importância na dispersão das espécies e na competição com os outros componentes do meroplâncton.

A presença das larvas de Decapoda no plâncton estuarino é de grande importância no ciclo de vida destas espécies, garantindo a colonização e o repovoamento do estoque parental, além de desempenharem papel importante na alimentação das larvas e 


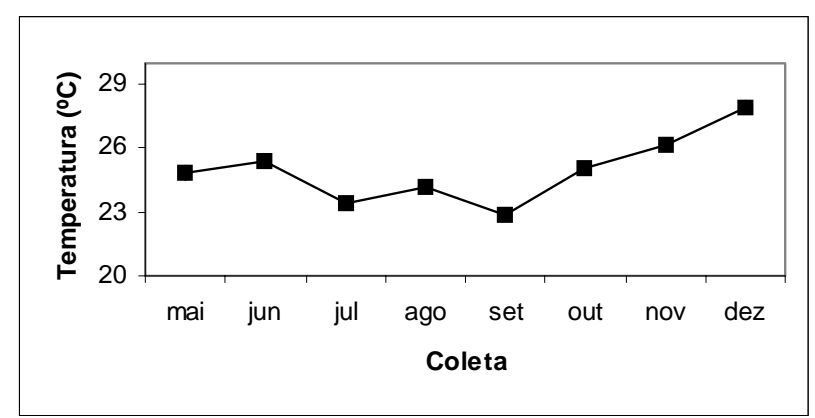

Figura 2 - Média mensal da temperatura no estuário do rio Piraquêaçú.

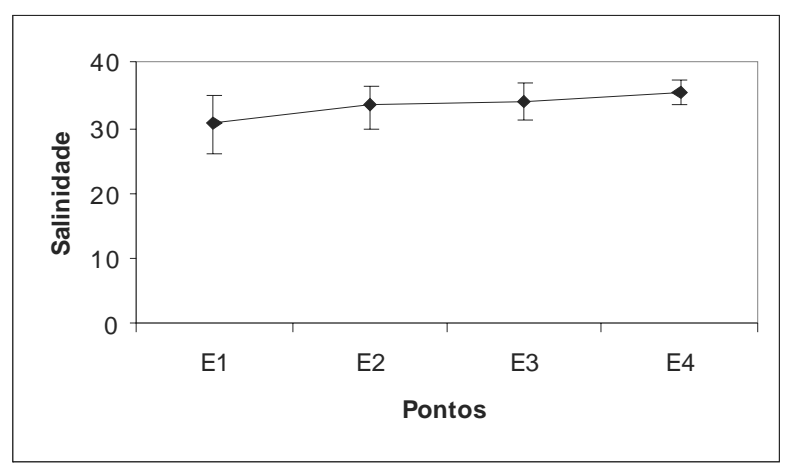

Figura 3 - Média da salinidade por pontos de amostragem no estuário do rio Piraquê-açú.

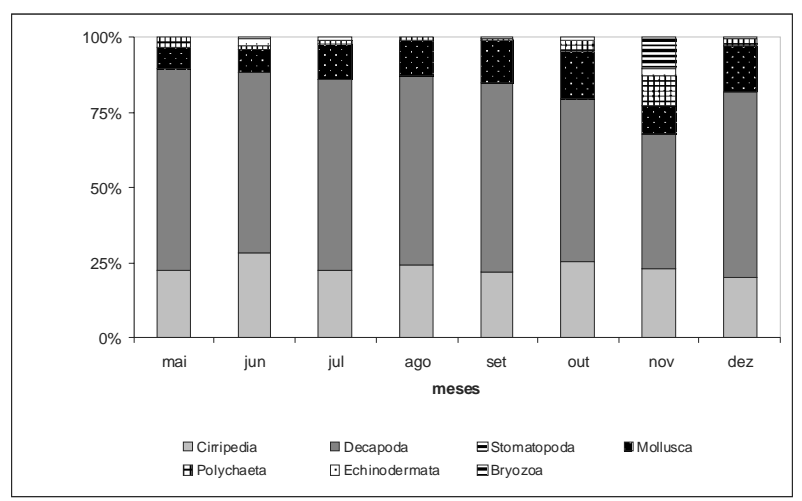

Figura 4 - Variações mensais na percentagem de abundância dos taxa componentes do meroplâncton no estuário do rio Piraquêaçú.

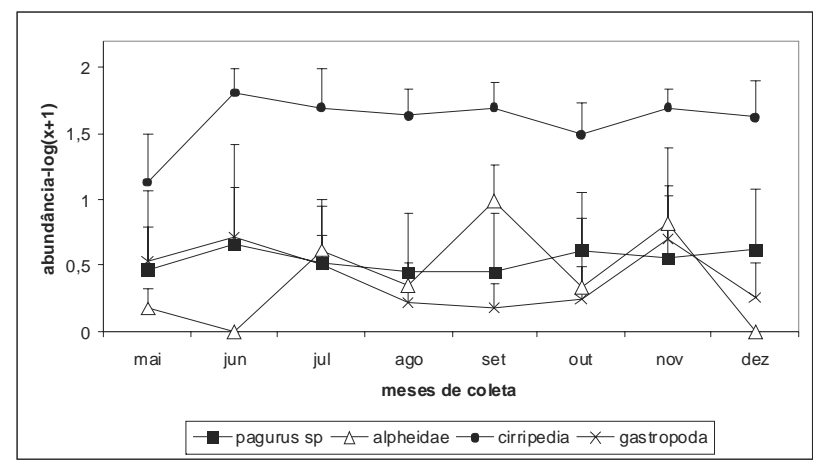

Figura 5 - Abundância média log $(x+1)$ dos principais componentes do meroplâncton do estuário do rio Piraquê-açú ao longo dos meses de amostragem. As barras de erro indicam o desvio padrão. juvenis de peixes de interesse comercial (Lindley, 1998; Brandini et al., 1997).

As larvas de Decapoda foram representadas pelos Brachyura, Anomura e Caridea. As larvas de Brachyura foram as mais abundantes em todos os meses, chegando a alcançar 500,41 ind. $\mathrm{m}^{-3}$ no mês de maio. Os Anomura, por sua vez, estiveram representados pelas famílias Paguridae e Diogenidae (ermitões), que alcançaram 52,35 ind. $\mathrm{m}^{-3}$ no mês de junho, sendo 49,82 ind. $\mathrm{m}^{-3}$ do gênero Pagurus, e Porcellanidae, com pico de abundância em maio com 14,33 ind. $\mathrm{m}^{-3}$. Os Caridea, principalmente da família Alpheidae, alcançaram 47,49 ind. $m^{-3}$ no mês de setembro. A maior freqüência e dominância de Brachyura dentro dos Decapoda também foi relatada em outros estudos como de Fernandes et al. (2002) na baía de Guanabara e de Sankarankutty et al. (1995) no estuário de Potengi, Natal.

Foram identificadas vinte espécies de Brachyura, pertencentes às famílias Grapsidae, Portunidae, Xanthidae, Ocypodidae e Pinnotheridae. Segundo Pohle et al. (1999), os representantes destas famílias possuem ocorrência comum em regiões estuarinas e de entre-marés em todo o Brasil. Destas espécies, Uca burgersi, Eurytium limosum, Pachygrapsus transversus, Metasesarma rubripes e Sesarma rectum foram as mais abundantes. S. rectum foi a espécie dominante em maio, com 231,70 ind. $\mathrm{m}^{-3}$, enquanto $U$. burgersi foi a mais representativa em julho, com 79,93 ind. $\mathrm{m}^{-3}$. M. rubripes e $P$. transversus dominaram em setembro com 36,82 e 35,16 ind. $\mathrm{m}^{-3}$, respectivamente. E. limosum apresentou seu maior valor de abundância em maio (71,15 ind. $\mathrm{m}^{-3}$ ) (Figura 6).

A maioria das espécies de Brachyura (16) foi encontrada nos meses mais quentes do período amostral. Montú (1980) relaciona a ocorrência de um

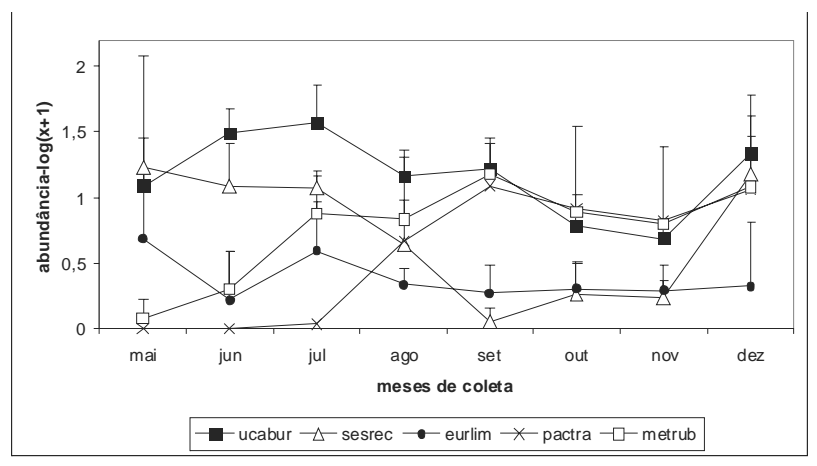

Figura 6 - Abundância média log $(x+1)$ das principais espécies de larvas de Decapoda Brachyura no estuário do rio Piraquê-açú ao longo dos meses de amostragem. ucabur - Uca burgersi; sesrecSesarma rectum; eurlim- Eurytium limosum; pactra- Pachygrapsus transversus; metrub- Metasesarma rubripes. As barras de erro indicam o desvio padrão. 
grande número de espécies nestes meses com a estratégia reprodutiva que as mesmas adotam de sincronizar suas atividades de desova com os meses de temperatura mais elevada.

O número de taxa de Brachyura encontrado neste estudo, entretanto, foi inferior ao registrado em outros estuários tropicais como no golfo de Tigullio (Itália), com 44 taxa (Pessani \& Salton, 1998), e na península Ibérica, com 30 taxa (González-Gordillo \& Rodríguez, 2003).

A abundância dos diferentes taxa apresentou diferenças significativas $(p<0,05)$ entre os meses de coleta para Brachyura (com maiores abundâncias em juIho e dezembro), Caridea (com maiores abundâncias em setembro e julho), Gastropoda (com maiores abundâncias em dezembro, julho e setembro) e Ophiuroidea, Polychaeta e Bryozoa (com maiores abundâncias em novembro) (Tabela 1). Todos estes grupos apresentaram abundâncias mínimas em maio, junho e julho, exceto Brachyura e Polychaeta.

A ANOVA sobre as abundâncias dos taxa nos diferentes pontos amostrais revelou que apenas Cirripedia, Ophiuroidea e Echinoidea apresentaram diferenças significativas $(p<0,05)$ (Tabela 2). Os náuplios de Cirripedia foram abundantes especialmente no ponto à montante e nos intermediários, sendo bastante tolerantes às variações de salinidade. Ophiuroidea e Echinoidea, com maiores abundâncias no ponto mais próximo à desembocadura, apresentaram-se mais sus- ceptíveis às salinidades baixas, já que são organismos tipicamente marinhos. González-Gordillo \& Rodríguez (2003) também encontraram um gradiente espacial de abundância e associaram tal fato à origem do estoque de adultos.

Os taxa encontrados se agruparam de acordo com a variação de abundância nos diferentes meses, o que pode refletir estratégias diferenciadas de ciclo de vida entre as espécies (Figura 7).

Das variáveis independentes testadas estatisticamente, a sazonalidade foi mais importante do que a distribuição espacial para a maioria dos taxa testados. Embora diferenças sazonais na estrutura da comunidade do zooplâncton sejam esperadas, principalmente em estuários subtropicais, muitas espécies mostram constância na sua ocorrência (Lopes, 1996). De acordo com Drake et al. (1998), em ambientes estuarinos tropicais, a variabilidade do plâncton obedece, preferencialmente, os ciclos nictemerais, lunares e intermareais.

Desta maneira, dentre os fatores que afetam os padrões de distribuição espacial e temporal do zooplâncton documentados por diversos autores (Lopes, 1996; Froneman, 2001; Rios-Jara, 1998), a temperatura mostrou ter maior influência na sazonalidade da abundância, e a salinidade mostrou ter maior associação com a distribuição espacial de algumas espécies, já que o estuário do Piraquê-açú apresentou um gradiente longitudinal de salinidade reduzido.

Tabela 1 - ANOVA da abundância (ind.m-3) dos componentes do meroplâncton ao longo dos meses (padrão temporal) do estuário do rio Piraquê-açú. Ns: não significante com a = 0,05.

\begin{tabular}{|c|c|c|c|}
\hline \multirow{2}{*}{$\begin{array}{l}\text { Taxa (variáveis } \\
\text { dependentes) }\end{array}$} & \multirow{2}{*}{$\begin{array}{l}\text { Abundância } \\
\text { média total } \\
\text { log }(x+1)\end{array}$} & $\begin{array}{c}\text { Variável } \\
\text { independente }\end{array}$ & \multirow[t]{2}{*}{ Grupos homogêneos } \\
\hline & & Mês & \\
\hline Cirripedia & $3,14 \pm 0,68$ & Ns & - \\
\hline \multicolumn{4}{|l|}{ Decapoda } \\
\hline Brachyura & $5,54 \pm 1,74$ & 0,043 & $\begin{array}{l}\min =\text { out, nov } \\
\max =\text { jul, dez }\end{array}$ \\
\hline $\begin{array}{l}\text { Paguridae e } \\
\text { Digenidae }\end{array}$ & $1,57 \pm 0,56$ & Ns & - \\
\hline Porcellanidae & $0,12 \pm 0,22$ & Ns & - \\
\hline Caridea & $0,69 \pm 0,4$ & 0,049 & $\begin{array}{l}\min =\text { jun, mai } \\
\max =\text { set, jul }\end{array}$ \\
\hline Stomatopoda & $\begin{array}{c}0,0098 \pm \\
0,041\end{array}$ & Ns & - \\
\hline \multicolumn{4}{|l|}{ Echinodermata } \\
\hline Ophiuroidea & $0,046 \pm 0,14$ & 0,001 & $\begin{array}{c}\min =\text { mai, jul, ago, set, } \\
\text { out, dez } \\
\text { max }=\text { nov }\end{array}$ \\
\hline Echinoidea & $0,032 \pm 0,18$ & Ns & - \\
\hline Polychaeta & $0,38 \pm 0,49$ & 0,000 & $\begin{array}{c}\min =\text { set, ago, jul, jun } \\
\text { max = nov }\end{array}$ \\
\hline Gastropoda & $1,48 \pm 0,61$ & 0,004 & $\begin{array}{c}\min =\text { mai, jun, nov } \\
\max =\text { dez, jul, set }\end{array}$ \\
\hline Bryozoa & $0,25 \pm 0,56$ & 0,000 & $\begin{array}{c}\min =\text { mai, ago, jun, set, } \\
\text { dez } \\
\max =\text { nov }\end{array}$ \\
\hline
\end{tabular}


Tabela 2 - ANOVA da abundância total dos componentes do meroplâncton nos pontos de amostragem (padrão espacial) do estuário do rio Piraquê-açú. Ns: não significante com a = 0,05.

\begin{tabular}{|c|c|c|}
\hline \multirow{2}{*}{$\begin{array}{c}\text { Taxa(variáveis } \\
\text { dependentes) }\end{array}$} & Variável independente & Grupos homogêneos \\
\hline & Ponto & Ponto (Duncan) \\
\hline Cirripedia & 0,001 & $\mathrm{E} 1, \mathrm{E} 2, \mathrm{E} 3>\mathrm{E} 4$ \\
\hline \multicolumn{3}{|l|}{ Decapoda } \\
\hline Brachyura & Ns & - \\
\hline $\begin{array}{l}\text { Paguridae e } \\
\text { Digenidae }\end{array}$ & Ns & - \\
\hline Porcellanidae & Ns & - \\
\hline Caridea & Ns & - \\
\hline Stomatopoda & Ns & - \\
\hline \multicolumn{3}{|l|}{ Echinodermata } \\
\hline Ophiuroidea & 0,001 & $\mathrm{E} 4, \mathrm{E} 2, \mathrm{E} 3>\mathrm{E} 1$ \\
\hline Echinoidea & 0,004 & $\mathrm{E} 4>\mathrm{E} 3, \mathrm{E} 2, \mathrm{E} 1$ \\
\hline Polychaeta & Ns & - \\
\hline Gastropoda & Ns & - \\
\hline Bryozoa & Ns & - \\
\hline
\end{tabular}

\section{CONCLUSÕES}

As larvas de Decapoda, de Cirripedia e de Gastropoda foram os principais componentes do meroplâncton no estuário do rio Piraquê-açú. Dentre os Decapoda, Brachyura foi o grupo dominante durante o período amostral, sendo representado por vinte espécies tipicamente costeiras e de regiões entre-marés. $O$ maior número destas espécies foi encontrada nos meses mais quentes do período amostral. A sazonalidade na abundância foi mais importante do que a distribuição espacial para a maioria dos taxa encontrados.

\section{AGRADECIMENTOS}

Ao Projeto RECOS Instituto do Milênio - MCT/ CNPq/PADCTIII pelo financiamento dos trabalhos e da bolsa de Iniciação Científica. Ao Departamento de Ecologia e Recursos Naturais pela infra-estrutura e aos colegas do Projeto pelo apoio durante as coletas de campo.

\section{REFERÊNCIAS}

Boschi, E.E. 1981. Larvas de Crustacea Decapoda. In: D. Boltovskoy (ed.) Atlas del Zooplancton del Atlantico Sudoccidental. INIDEP. 699-758pp.

Boschi, E.E. 1996. Larvas de Crustacea Decapoda. In: R. Gasca, \& E.S. Morales (eds.) Introducion al estudio del zooplancton marino. El Colegio de la Frontera Sur (ECOSUR)/CONACYT. 343-374pp.

Brandini, F.P.; Lopes, R.M.; Gutseit, K.S.; Spach, H.I. \& Saffi, R. 1997. Planctonologia na plataforma continental do Brasil: diagnose e revisão bibliográfica. $1^{\circ}$ Edição. FEMAR, Paraná, 196p.

Drake, P.; Arias, A.M. \& Rodríguez, A. 1998. Seasonal and tidal abundance patterns of decapod crustacean

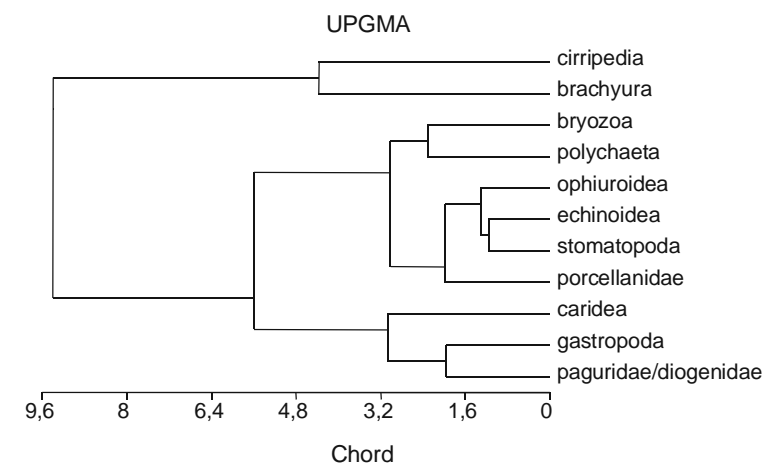

Figura 7 - Dendograma da análise de agrupamento dos taxa através da distância Chord com o método de UPGMA.

larvae in a shallow inlet (SW Spain). Journal of Plankton Research. 20(3):585-601.

Fernandes, L. D.; Bonecker, S.L.C \& Valentin, J.L.A. 2002. A Dynamic of Decapod Crustacean Larvae on the entrance of Guanabara Bay. Brazilian Archives of Biology and Technology. 45(4):491-498.

Froneman, P.W. 2001. Seasonal Changes in Zooplankton Biomass and Grazing in a Temperate Estuary, South Africa. Estuarine, Coastal and Shelf Science. 52:543-553.

González-Gordillo, J.I. \& Rodríguez, A. 2003. Comparative seasonal and spatial distribution of decapod larvae assemblages in three coastal zones of the south-western Iberian Peninsula. Acta Oecologica. 24:S219-S233.

Gurney, R. 1942. Larvae of Decapod Crustacea. Bernard Quaritch, London, 188p.

Kennish, M.J. \& Lutz, P.L. 1995. Ecology of Marine Invertebrate Larvae. CRC Press, Boca Raton, 464p.

Lindley, J.A. 1998. Diversity, biomassa, and production of decapod crustacean larvae in a changing environment. Invertebrate Reproduction and Development. 33(2-3):209-219.

Lopes, R.M. 1996. Hydrography and zooplankton community structure: a comparative study among estuaries of the Juréia-Itatins ecological station (southeastern Brazil). Nerítica. 10:7-26.

Montú, M. 1980. Zooplâncton do estuário da Lagoa dos Patos: estrutura e variações espaciais e temporais da comunidade. Atlântica. 4:53-72.

Omori, M. \& Ikeda, T. 1992. Methods in Marine Zooplankton Ecology, Krieger Plubishing Company, Florida, 329p.

Pessani, D. \& Salton L. 1998. Planktonic larval stages of Brachyura in the Gulf of Tigullio (Ligurian Sea, Italy). Invertebrate Reproduction and Development. 33(2-3):201-208. 
Pohle, G.; Mantelatto, F.L.; Negreiros-Fransozo, M.L. \& Fransozo, A. 1999. Larval Decapoda (Brachyura). In: D. Boltovskoy (ed.) South Atlantic Zooplankton. Leiden. 1281-1351pp.

Rios-Jara, E. 1998. Spatial and Temporal Variations in the Zooplankton Community of Phosphorescent Bay, Puerto Rico. Estuarine, Coastal and Shelf Science. 46:797-809.

Sankarankutty, C.; Oliveira, J.E.L.; Cunha, K.M.F.; Silva, A.C.C. \& Barroca, E.V.G. 1995. Further observations on zooplankton of the Potengi estuary
(Natal, Rio Grande do Norte) with special reference to the larvae of Brachyura (Crustacea, Decapoda). Revista Brasileira de Zoologia. 12(2):273-301.

Smith, D.L. \& Johnson, K.B. 1997. A guide to marine coastal plankton and marine invertebrate larvae. Kendall/Hunt Publishing Company, Debuque, 127p.

Sterza, J.M. 2002. Composição, abundância e distribuição espaço-temporal do zooplâncton relacionadas com a hidroquímica no sistema estuarino baía de Vitória/canal da Passagem, Vitória, ES. Dissertação de Mestrado. Universidade Estadual do Norte Fluminense. 86p. 